\section{A case of streptococcal surgical site infection following Mohs surgery}

\author{
Kami B. Lowery, John J. Kohorst, \\ C. Helen Malone, Ikue Shimizu \\ Department of Dermatology, Baylor \\ College of Medicine, 1 Baylor Plaza, \\ Houston, TX, USA
}

\begin{abstract}
Group A $\beta$-hemolytic Streptococcal (GAS) cellulitis is an uncommon surgical site infection that presents with rapid onset of pain and swelling in the first few days after a procedure. Unlike staphylococcal cellulitis, GAS cellulitis lacks purulence and spreading erythema. The absence of these classic signs may delay the diagnosis of GAS cellulitis and lead to severe complications. We present the case of an immunosuppressed 49-year-old patient who developed swelling and severe pain at his incision site two days after undergoing Mohs micrographic surgery on his forehead. He was clinically diagnosed with GAS cellulitis and recovered with intravenous antibiotics. Unfortunately, there is a paucity of information about GAS cellulitis in the dermatologic literature and clinicians need to recognize and aggressively treat this rare but serious complication of Mohs micrographic surgery.
\end{abstract}

\section{Introduction}

Surgical Site Infections (SSI), although low in prevalence, are the most common adverse event in patients undergoing Mohs micrographic surgery. ${ }^{1}$ Patients may experience fever and local symptoms such as redness, pain, warmth, swelling, and drainage of pus or cloudy fluid at the wound site within several days of their procedure. ${ }^{2}$ Dermatologic SSIs typically result from microorganisms present on the skin at the incision site, most commonly Staphylococcus aureus (S. aureus) or streptococcal bacteria. ${ }^{2}$ We present an atypical case of surgical site infection in an immunosuppressed patient who underwent Mohs micrographic surgery for treatment of a squamous cell carcinoma.

\section{Case Report}

A 49-year-old man with a history of a renal transplant eight years prior on tacrolimus and mycophenolate mofetil presented for management of a biopsy proven squamous cell carcinoma on the right temple. Mohs micrographic surgery was performed. The malignancy was cleared in three stages and the post-operative size was $1.8 \mathrm{~cm} \times 1.4 \mathrm{~cm}$ (Figure 1). The defect was closed primarily in a complex layered fashion (Figure 2). A standard dressing was applied, and the patient received wound care instructions.

Two days following surgery, the patient returned to the dermatology clinic with severe pain and mild swelling surrounding his surgical site (Figure 3). He denied drainage from the incision, sensation of pressure, vision changes, pain with eye movement, or systemic symptoms such as subjective fever or chills. On exam, the patient was afebrile. He had mild swelling and blanching erythema surrounding the surgical site. Tenderness to palpation was noted. No warmth, fluctuance or crepitus was observed.

Although the patient's clinical exam was relatively unimpressive, his rapid symptom progression and severe pain were concerning for an aggressive streptococcal soft tissue infection requiring intravenous antibiotics. Labs and imaging were ordered. ENT was consulted should surgical intervention become necessary. The patient was transferred to the emergency department then admitted to internal medicine. Broadspectrum intravenous antibiotics were started including linezolid for MethicillinResistant Staphylococcus Aureus (MRSA) coverage (the patient reported vancomycin allergy), ceftriaxone, and metronidazole. Laboratory studies revealed a mild leukocytosis $(12,500 \mathrm{WBCs})$. Two blood cultures showed no growth. A CT maxillofacial without contrast (due to the patient's renal transplant) confirmed extensive right periorbital, cheek, and scalp tissue swelling suspicious for cellulitis. No drainable fluid collection, hematoma or abscess formation was noted.

On inpatient day two, the patient's symptoms improved. Ophthalmology, consulted due to concern for orbital cellulitis, determined that the patient more likely had periorbital cellulitis. ENT determined debridement was unnecessary. Infectious Disease evaluated the patient and suspected a streptococcal infection due to the rapidity of onset after Mohs surgery. As the responsible organism was likely skin-derived, antibiotics were narrowed to IV Linezolid alone for gram-positive and MRSA coverage. Prior to discharge, a small amount of thin, cloudy fluid was expressed from the suture line and sent for gram stain and cul-
Correspondence: Kami Lowery, BCM 903 , Baylor College of Medicine, 7200 Cambridge St, Houston, TX 77030, USA

E-mail: kami.lowery20@gmail.com

Tel.: +1 832-472-9763

Key words: Streptococcus; cellulitis; Mohs surgery; infection.

Conflict of interest: the authors declare no potential conflict of interests.

Received for publication: 31 July 2020.

Accepted for publication: 12 August 2020.

This work is licensed under a Creative Commons Attribution-NonCommercial 4.0 International License (CC BY-NC 4.0).

(C) Copyright: the Author(s), 2020

Licensee PAGEPress, Italy

Dermatology Reports 2020; 12:8819

doi:10.4081/dr.2020.8819

ture. Rare to few gram-positive cocci in pairs were seen and a moderate amount of Methicillin-Sensitive Staphylococcus Aureus (MSSA) was isolated.

On the day of discharge, the patient noted significantly decreased surgical site pain and erythema. He was discharged to complete a ten-day course of oral Linezolid (Figure 4).

\section{Discussion}

This case illustrates a rare complication of Mohs micrographic surgery and the need for a high index of suspicion for non-purulent Group A $\beta$-hemolytic Streptococcal (GAS) cellulitis in early postoperative patients with acute pain and edema in the early postoperative period lacking the classic findings of purulent Surgical Site Infection (SSI).

SSIs are the most frequent complication of Mohs micrographic surgery. Though typically performed with a clean rather than sterile technique, studies have demonstrated low rates of SSI in patients undergoing Mohs surgery around $0.4 \% .{ }^{1} S$. aureus is the most common cause of surgical site infection in approximately $20 \%$ of cases and many studies have examined the risk factors and preventative measures focusing on eradication of staphylococcus pre- and perioperatively. ${ }^{3-4}$ In contrast, GAS has been shown to be the causative pathogen in only approximately $1 \%$ of surgical site infection cases and has received minimal focus in the dermatologic literature. ${ }^{4}$ Other specialties such as plastic surgery have published more 
frequently on the presentation and treatment of postoperative GAS infection. . $^{5}$

GAS cellulitis differs from classic staphylococcal cellulitis in clinical presen- tation. While classic staphylococcal SSI presents with delayed pain, swelling, erythema, purulent exudate and systemic symptoms 4-8 days after surgery, GAS cel-

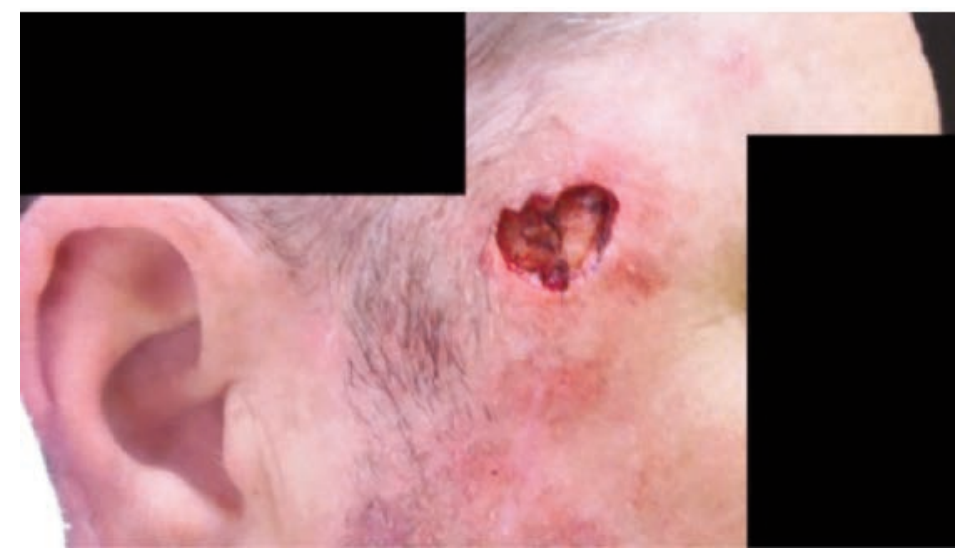

Figure 1. Postoperative defect of the right temple immediately following Mohs surgery.

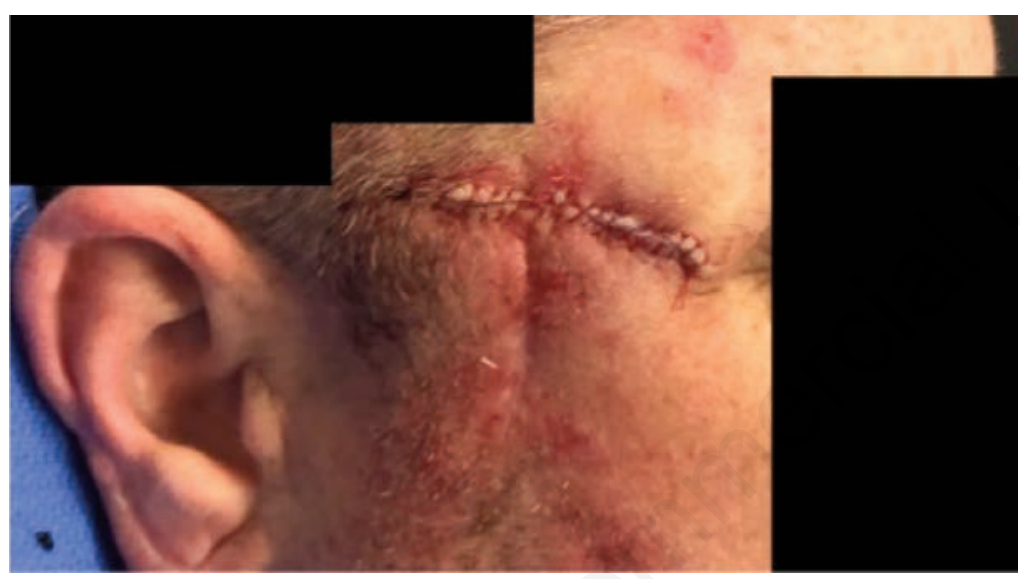

Figure 2. Surgical site immediately following complex linear closure. lulitis demonstrates rapidly progressive pain and swelling in the first few days following surgery but typically lacks exudate and the classic expanding erythema. ${ }^{5}$ The subtle clinical features of GAS cellulitis are easily missed. If treatment is delayed, GAS infections may evolve into necrotizing fasciitis with diffuse bullae, widespread skin necrosis, and sepsis with multiorgan failure. $^{5}$

GAS cellulitis was suspected in our immunosuppressed patient based on the clinical presentation of severe surgical site pain and swelling within 48 hours of surgery. He was treated immediately and aggressively with intravenous antibiotics and consultation for wound debridement to contain the infection. In general, treatment of rapidly progressive GAS infections with concern for necrotizing fasciitis consists of IV antibiotics and early surgical intervention with or without hyperbaric oxygen to contain infections before widespread necrosis. ${ }^{5}$ Fortunately, our patient made a rapid recovery with reduction in surgical site pain and swelling during his hospital course.

\section{Conclusion}

Ultimately, GAS was not identified by blood or swab culture in this patient. In general, the inability to culture the causative organism in a surgical site infection is common. ${ }^{4}$ MSSA was isolated late in the patient's hospitalization but was presumed to be a contaminant.

In conclusion, this case highlights the importance of a high index of suspicion for surgical site infection in patients with acute

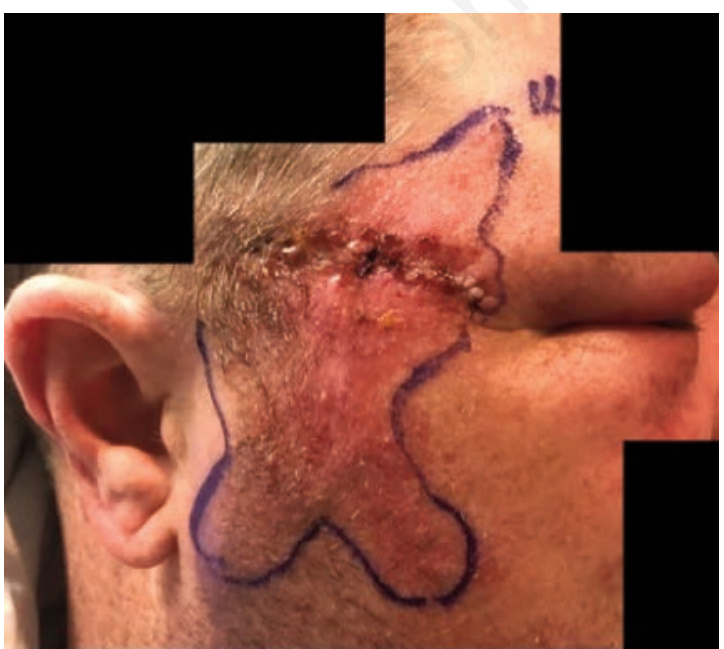

Figure 3. Two days following Mohs surgery and reconstruction with diffuse and progressive swelling, erythema and surgical site pain. All clinically evident erythema was demarcated with a blue pen.

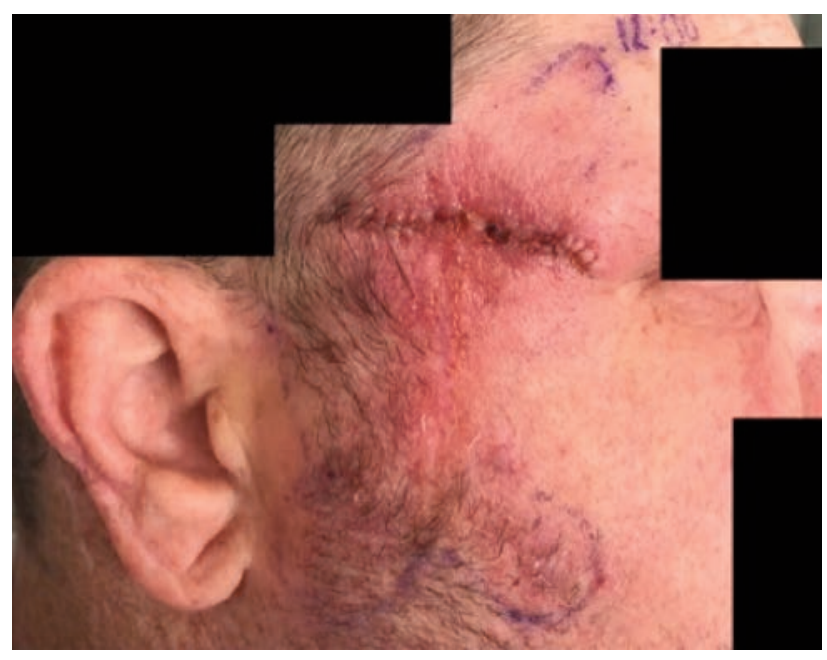

Figure 4. Day of discharge (four days postoperative) with decreased surgical site pain and erythema. 
pain and swelling in the early postoperative period. Though an uncommon cause of postoperative infection, GAS infections present sooner, more aggressively and without the classic signs of purulent staphylococcal cellulitis and must be treated aggressively.

\section{References}

1. Alam M, Ibrahim O, Nodzenski M, et al. Adverse events associated with Mohs micrographic surgery: multicen- ter prospective cohort study of 20821 cases at 23 centers. JAMA Dermatol 2013;149:1378-85.

2. Rosengren H, Heal C, Smith S. An update on antibiotic prophylaxis in dermatologic surgery. Curr Derm Rep 2012;1:55-63.

3. Saleh K, Schmidtchen A. Surgical site infections in dermatologic surgery: etiology, pathogenesis, and current preventative measures. Dermatol Surg. 2015;41:537-49.

4. National Nosocomial Infections Surveillance (NNIS) report, data sum- mary from October 1986-April 1996 issued May 1996. A report from the National Nosocomial Infections Surveillance (NNIS) system. Am J Infect Control 1996;24:380-8.

5. Goldberg R, Li T. Postoperative infection with group A beta-hemolytic streptococcus after blepharoplasty. Am J Opthalmol 2002;134:908-10.

6. Gonzáles Alana I, Marin de la Cruz D, Palao Doménech R, Barret Nerin J. Necrotizing fasciitis after liposuction. Acta Chir Plast 2007;49:99-102. 